Ann. Zootech., I978, 27 (I), I-7.

\title{
Intérêt du rationnement du caneton mâle de Barbarie entre les âges de 8 et 12 semaines
}

\author{
B. LFCLERCQ et H. de CARVILI,E \\ Station de Recherches avicoles, \\ Centre de Recherches de Tours, I.N.R.A., \\ Nouzilly-37380 Monnaie (France)
}

\begin{abstract}
Résumé
Deux expériences ont été réalisées, portant chacune sur Ioo canetons de Barbarie mâles. La moitié des animaux a été rationnée à partir de l'âge de 8 semaines. Dans chaque lot, un animal sur deux est sacrifié à ro semaines, 1'autre à 12 semaines. Lors de la première expérience, le rationnement est faible (de l'ordre de $5 \mathrm{p}$. cent en moyenne), dans la seconde, il est plus sévère (environ 20 p. cent).

Le rationnement faible n'altère pas la croissance du caneton et tend à améliorer légèrement son indice de consommation. Le rationnement sévère ralentit significativement la croissance et conduit à une détérioration de l'indice de consommation. Le retard de croissance correspond en majeure partie à une réduction des lipides de réserves. Le poids de la cuisse et celui des masses musculaires pectorales sont très peu diminués en valeur absolue; leur proportion dans la carcasse n'est pas influencée par le rationnement, mais seulement par l'âge des animaux.
\end{abstract}

\section{Introduction}

L'abattage du caneton de Barbarie ne doit pas survenir trop précocement. Le développement des masses musculaires pectorales intervient en effet en fin de croissance pour donner des carcasses de mieux en mieux pourvues de parties comestibles à mesure que l'animal vieillit (LECLERCQ et de CARVILIE, I975, LECLERCQ et de CARVII,LE, I976). Malheureusement, ce phénomène est accompagné d'un ralentissement notable de la croissance et d'une dégradation rapide de l'indice de consommation. Les deux expériences présentées ci-dessous ont été entreprises afin de savoir si un rationnement imposé aux canetons en fin de période d'élevage peut limiter les dépenses d'aliment tout en permettant le développement normal des masses musculaires. Nous nous sommes intéressés au caneton mâle chez lequel des économies d'aliment sont particulièrement souhaitables pour trois raisons essentielles :

I. Le développement des muscles pectoraux est plus tardif chez lui que chez la femelle. 
2. La consommation alimentaire du mâle est plus élevée du fait du dimorphisme sexuel important.

3. Sa carcasse trop lourde se commercialise moins bien que celle de la femelle; un ralentissement de la croissance sans détérioration de la qualité de carcasse ne pourrait qu'être bénéfique à sa commercialisation.

\section{Matériel et méthodes}

Au cours de chacune des expériences, ioo canetons mâles (Grimaud R 32) ont été répartis dans ro cases de ro sujets. Ces cases ont roo $\mathrm{cm}$ de largeur et I $60 \mathrm{~cm}$ de longueur et sont équipées d'un sol grillagé (maille carrée de $2 \mathrm{~cm}$ de

\section{TABIEAU I}

Infuence du rationnement sur la croissance et la consommation du caneton male de Barbarie

(Expérience $I$ )

Effect of feed restriction on growth and feed intake of muscovy ducklings

(Experiment I)

Poids vif à 8 semaines

Live weight at 8 weeks

Poids vif à Io semaines

Live weight at to weeks

Gain de poids 8-ro semaines

Weight gain 8-ro weeks

Consommation 8-10 semaines (g) ...........

Feed intake 8-10 weeks

Indice de consommation 8-1o semaines.

Feed conversion ratio 8 - 10 weeks

Indice de consommation 0 - Io semaines.

Feed conversion ratio o-to weeks

Poids vif à Io semaines $(\mathrm{g})$

Live weight at $\mathrm{To}$ weeks

Poids vif à $\mathbf{r} 2$ semaines

Live weight at I2 weeks

Gain de poids IO-I 2 semaines $(g)(* *) . . . . . .$.

Weight gain IO-I2 weeks

Consommation Io-I 2 semaines

Feed intake IO-I2 weeks

Indice de consommation Io- I'2 semaines . . . . . .

Feed conversion ratio $10-12$ weeks

Indice de consommation 0 -I 2 semaines.

Feed conversion ratio $0-12$ weeks
Lot rationné $\left({ }^{*}\right)$

(Restricted)

Lot témoin (

(Control ad libitum)

3008

3505

497

2784

5,60

$5,3 \mathrm{I}$

2,99

2,95

3467

3486

3710

3728

242

2742

2852

I I, 74

$1 \mathrm{I}, 33$

$3,5^{6}$

3,49

$\left(^{*}\right)$ Tous les animaux ont été nourris à volonté jusqu'à l'âge de 8 semaines. All the ducklings iccre fed ad-libitum until 8 weeks of age.

$\left.{ }^{* *}\right)$ Sur les animaux conservés au-delà de ro semaines. Only birds kept after Io weeks of age. 
côté). Les animaux sont éclairés 24 heures par jour dès la naissance. La durée d'éclairement est réduite à Io heures par jour à l'âge de 2 semaines.

Les animaux sont nourris à volonté jusqu'à l'âge de 8 semaines avec un régime couvrant largement leurs besoins (LECLERCQ et de CARVILLE, 1976). A cet âge, les animaux de 5 cases sont rationnés quotidiennement tandis que les 5 autres cases continuent d'être alimentées à volonté. Le régime distribué est composé de $44 \mathrm{p}$. cent de maïs, 40,5 p. cent de blé, $8 \mathrm{p}$. cent de tourteau de soja (49 p. cent M.A.T.), 5 p. cent de farine de viande (5o p. cent M.A.'T.), o,4 p. cent de sel, I p. cent de carbonate de calcium, $0,5 \mathrm{p}$. Ioo de phosphate bicalcique, $0,6 \mathrm{p}$. cent d'un mélange de vitamines et oligominéraux selon la composition indiquée précédemment (LECLERCQ et de CARVIL, $\mathrm{E}$, I976). Ce régime renferme approximativement 2 goo Kcal $/ \mathrm{kg}$ et exactement $\mathrm{I}_{5,2}$ p. cent de M.A.T. $(\mathrm{N} \times 6,25)$.

Le rationnement a lieu de l'âge de 8 semaines jusqu'à celui de $\mathbf{2}$ semaines. Il consiste à distribuer chaque jour une quantité limitée d'aliment. Celle-ci est

\section{TABLEAU 2}

Infuence du rationnement sur la croissance et la consommation du caneton male de barbarie

(Expévience 2)

Eflect of feed restriction on growth and feed intake of muscovy ducklings (Experiment 2)

Poids vif à 8 semaines ( Live weight at 8 weeks

Poids vif à Io semaines (g)

. . . . . . . . .

Lot témoin $\left(^{*}\right)$

Lot restreint (*) (Control ad libitum) (Restricted)

Live weight at Io weeks

Gain de poids 8 - Io semaines (g)

Weight gain 8 -Io weeks

Consommation 8-10 semaines (g)

Feed intake 8-ro weeks

Indice de consommation 8-10 semaines.

lieed conversion ratio 8-10 teeks

Indice de consommation o-ro semaines. . .....

lieed conversion ratio $0-10$ weeks

Poids vif à Io semaines $(\mathrm{g})\left({ }^{* *}\right)$. . . . . . . . . .

Live weight at to weeks.

Poids vif à 12 semaines

Live weight at 12 weeks

Gain de poids Io-I2 semaines (g) (**)

(g) $(* *)$

Weight gain Io-I2 weeks

Consommation I $0-12$ semaines

Feed intake Io-I2 weeks

Indice de consommation ro-12 semaines

Feed conversion ratio ro-12 wecks

Indice de consommation o-12 semaines.

Feed conversion ratio o-I2 weehs

2779

3206

3297

028

2710

4,32

2,90

$3 \cdot 47$

3629

382

2718

$7, \mathrm{I}_{2}$

3,35
3453 (***) $^{*}$

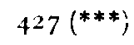

$2370(* * *)$

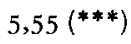

$2,9 \mathrm{I}$

3219

234 (***)

2075 (***) $^{* *}$

$8,87(* * *)$

$3,3^{8}$

(*) Tous les animaux ont été nourris à volonté jusqu'à l'âge de 8 semaines. All the ducklings ieterc fed ad-libitum until 8 weeks of age.

(*) Sur les animaux conservés au-delà de ro semaines. Only birds kept after Io weeks of age.

(***) Différence significative au seuil 5 p. cent. Significant difference at 0.05 level. 
calculée de façon à ne représenter qu'un certain pourcentage de la consommation de la semaine précédente des lots nourris à volonté. Ce décalage d'une semaine modifie un peu le rationnement réellement effectué par rapport à celui qui était prévu. Quoi qu'il en soit, celui-là peut être calculé à partir des quantités d'aliment ingérées par les canetons (tabl. I et 2).

Sur les carcasses on procède aux mesures suivantes : poids du filet et poids de la cuisse. De plus, dans la deuxième expérience, à l'âge de 12 semaines, Io ani maux rationnés et $I 0$ nourris à volonté sont broyés afin de procéder à la détermination des lipides totaux de la carcasse. Ces animaux sont choisis de façon que leur poids moyen soit semblable à la moyenne de leur lot.

Les deux expériences ne diffèrent que par l'intensité du rationnement imposé: faible dans la première expérience, plus prononcé dans la seconde. Les deux expériences se sont déroulées en hiver. Dans les deux cas la température des locaux d'élevage oscillait entre $3^{0}$ et $12^{\circ}$ avec pour moyenne $7^{0}$ dans la première et $6^{0}$ dans la seconde.

Les animaux sont toujours pesés individuellement après $I_{7}$ heures de jeûne total. Ils sont identifiés par une bague d'aile, ce qui permet de calculer les gains de poids vif par individu.

\section{Résultats}

Les résultats crincernant la croissance et la consommation d'aliment enregistrés au cours de l'expérience I font l'objet du tableau $\mathbf{r}$. La restriction alimentaire imposée aux animaux rationnés est modérée : 94 p. cent de la consommation des témoins entre 8 et ro semaines, $96 \mathrm{p}$. cent entre ro et $\mathrm{r} 2$ semaines. La croissance est très légèrement ralentie entre 8 et ro semaines et non modifiée entre Io et 2 semaines. I 'indice de consommation observé au cours de ces deux périodes est le plus souvent à l'avantage du lot restreint. L'indice global (o à Io semaines ou $o$ à I2 semaines) est toujours à l'avantage du lot restreint bien que la différence ne soit pas significative. Les mesures effectuées lors de 1'abattage et présentées dans le tablean 3 ne révèlent aucune différence significative entre les deux lots de canetons.

Nous rassemblons dans le tableau 2 les résultats de la seconde expérience concernant la croissance et la consommation d'aliment. Cette fois la restriction est plus sévère puisque entre $S$ et io semaines le lot restreint ne consomme que 87,5 p. cent de ce qu'ingère le lot nourri à volonté; entre Io et I 2 semaines, ce niveau d'ingestion devient $76,4 \mathrm{p}$. cent du lot témoin. La croissance est alors significativement ralentie au cours de ces deux périodes et les indices de consommation correspondant sont toujours en défaveur des canetons rationnés. L'indice de consommation total (o à Io semaines ou o à $\mathrm{I} 2$ semaines) est un peu plus élevé chez les animaux restreints. Les mesures effectuées sur les carcasses sont rassemblées dans le tableau 3. Le rationnement réduit significativement le poids des carcasses, du filet et de la cuisse aux deux âges d'abattage. De plus, à I 2 semaines, la teneur en lipides de la carcasse non éviscérée est significativement réduite par le rationnement. A Io semaines, on observe aussi que le rendement à l'abattage est meilleur che\% les canetons nourris à volonté, avantage qui n'apparaît plus à $\mathrm{r} 2$ semaines. Enfin, 1'analyse de covariance du poids du filet et de celui de la cuisse en fonction du poids vif montre que l'effet du régime n'est pas significatif, seul celui de l'âge le demeure. 


\begin{tabular}{|c|c|c|c|c|c|c|}
\hline & 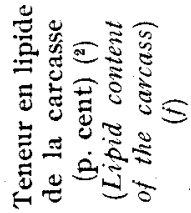 & 11 & 11 & 11 & 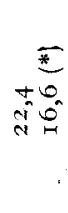 & 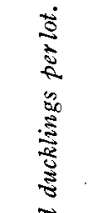 \\
\hline$\Xi$ & 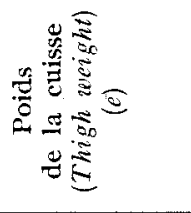 & 尽会 & 式 & $\underbrace{\mathbb{E}}_{\substack{\infty \\
0}}$ & 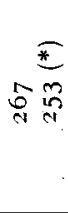 & 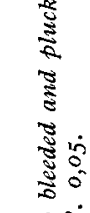 \\
\hline 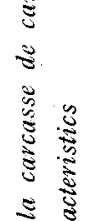 & 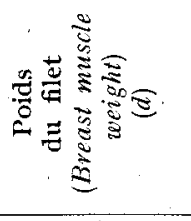 & $\mathbb{C}_{\mathbb{1}}^{\infty}$ & बis & 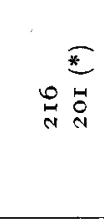 & 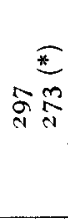 & 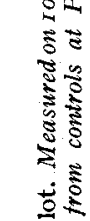 \\
\hline 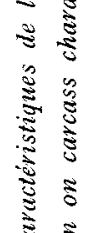 & 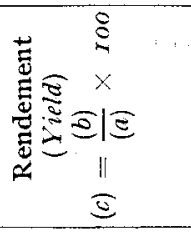 & 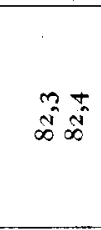 & $\begin{array}{c}\text { mo } \\
\text { ŝ } \\
\infty\end{array}$ & $\underbrace{2}$ & $\begin{array}{ll}0 & 0 \\
01 & m \\
\infty & \infty\end{array}$ & 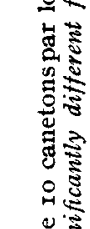 \\
\hline 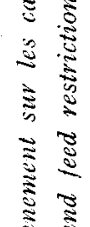 & 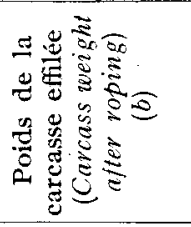 & $\begin{array}{ll}0 & 1 \\
\infty & 0 \\
0 & 0 \\
1 & 1\end{array}$ & $\begin{array}{ll}\infty & n \\
0 & n \\
0 & 0 \\
m & n\end{array}$ & 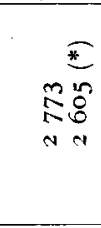 & 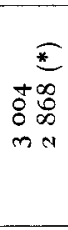 & 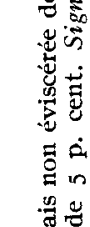 \\
\hline 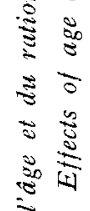 & 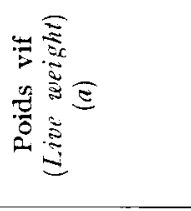 & $\begin{array}{l}\infty \\
\infty \\
+\infty \\
m \\
m\end{array}$ & $\begin{array}{l}n \mathbb{N} \\
\stackrel{N}{N} \\
m \\
m\end{array}$ & 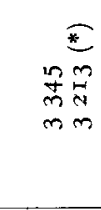 & 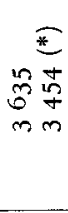 & 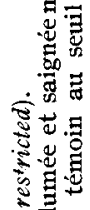 \\
\hline 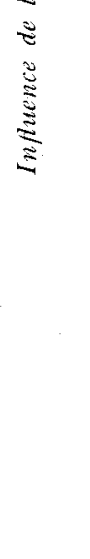 & : & 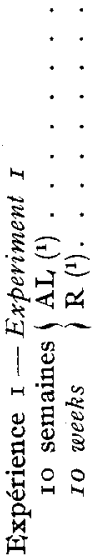 & 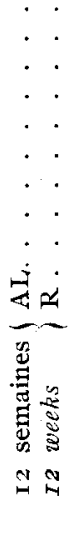 & 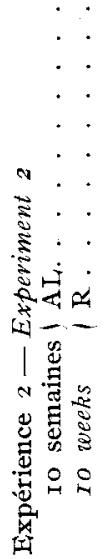 & 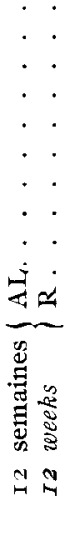 & 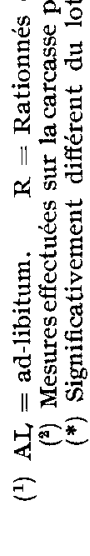 \\
\hline
\end{tabular}




\section{Discussion et conclusion}

Le caneton de Barbarie mâle supporte un rationnement léger ( $5 \mathrm{p}$. cent) sans guère modifier sa croissance et en améliorant un peu son indice de consommation. Un rationnement plus sévère conduit inéluctablement à un retard de croissance appréciable. De ce point de vue, le caneton de Barbarie réagit donc comme le poulet (Auckiand et Fur,on, I973; Simon et al., 1977) et le canard de Pékin (AUCKLAND, I973). Toutefois, contrairement à ces deux espèces, il réduit dans de larges proportions ses réserves adipeuses. En effet, la différence de lipides totaux est de l'ordre de $200 \mathrm{~g}$ pour une différence de poids vif qui est du même ordre. Le retard de croissance correspond donc pour sa majeure part à un ralentissement des dépôts adipeux. Ce n'est cependant pas la seule explication puisque le développement des muscles pectoraux et de la cuisse est un peu ralenti, lui aussi. Toutefois, la diminution de ces masses anatomiques ne fait que refléter le développement corporel puisque l'analyse de covariance avec le poids vif comme variable explicative se traduit par un effet nul des régimes.

Enfin, une fois de plus nous mettons en évidence l'importance de l'âge sur la proportion de muscles pectoraux de la carcasse. Les dissections effectuées sur 8 lots de 25 animaux sacrifiés à Io ou I $z$ semaines montrent qu'en moyenne entre ces âges le développement des filets représente $53,8 \mathrm{p}$. cent du gain de poids vif, que les animaux soient rationnés $(54,2$ et 59,8 p. cent dans les expériences I et 2$)$ ou nourris à volonté ( 45,2 et $55,9 \mathrm{p}$. cent dans chacune des expériences).

En définitive, le rationnement modéré $d u$ caneton de Barbarie peut être conseillé. S'il est un peu sévère (plus de $5 \mathrm{p}$. cent) il présente le seul inconvénient d'augmenter légèrement 1 'indice partiel de consommation. La carcasse est cependant nettement moins grasse et devrait alors être mieux valorisée commercialement.

Accepté pour publication en septembre 1977.

\section{Summary \\ Interest of restricted feeding of male Muscovy ducklings from 8 to $\mathrm{I} 2$ weeks of age}

Two experiments, each involving roo male Muscovy ducklings, were carried out. Half of the birds were fed restricted amounts of food from 8 weeks of age. In each group every second duckling was sacrificed at the age of 10 weeks and the other one at 12 weeks. The feed allowance was slightly restricted (about $5 \mathrm{p}$. cent on an average) in the first experiment and severely in the second one (about 20 p. cent).

A slight feed restriction did not affect growth, but tended to slightly improve feed conversion ratio. Severe restriction significantly reduced growth rate and led to a poorer feed conversion ratio. Delayed growth mainly corresponded to a reduction of lipid reserves. Weights of thighs and breast muscles decreased very little in absolute values; their proportion in the carcass was not influenced by the feed restriction, but only by the age of the ducklings. 


\section{Références bibliographiques}

AvCkLAND J. N., I973. Fffect of feeding restricted amounts of a medium and a high protein diet on growth and body composition of ducklings. J. Sci. Fd Agric., 24, 719-726.

AUCKLAND J. N., FULTON R. B., 1973. Effect of feeding restricted amounts of a medium and a high protein diet during the finishing period on growth, fat deposition and feed efficiency or male and female broilers. J. Sci. Fd Agric., 24, 709-7I 7 .

Lecherco B., Carvilite H. (de), I975. Besoin en protéines du caneton de Batbarie entre les âges de 4 et I semaines. Ann. Zootech., 24, 2 I 7-227.

IFCLERCQ B., CARVIrLE H. (de), I976. L'alimentation azotée du caneton de Barbarie : étude du besoin du caneton mâle entre les âges de 4 et I2 semaines. Ann. Zootech., 25, I89-I97.

Simon J., Zybio A., Guil.aume J., Buvm J. C., i977. Recherche d'une limitation de l'engraissement du poulet de chair par un léger rationnement alimentaire entre 6 et 8 semaines. Arch. Gefingelk., 41, sous presse. 